\title{
PRESCRIÇÃO DE ENFERMAGEM NO CENTRO DE TERAPIA INTENSIVA: ESTUDO COMPARATIVO DAS OPINIÕES DOS ENFERMEIROS E PESSOAL AUXILIAR DE ENFERMAGEM
}

\author{
Adriana Katia Corrêa ** \\ Jader Naves dos Reis ***
}

\begin{abstract}
RESUMO - Refletindo a prática em um Centro de Terapia Intensiva, nós indagamos se a prescrição de enfermagem vem realmente coordenando as ações de enfermagem efetuadas pelo pessoal auxiliar. Com o objetico principal de conhecer e confrontar as opiniões dos enfermeiros e do pessoal auxiliar quanto ao conteúdo da prescrição de enfermagem, 'aplicamos um questionário a 5 enfermeiros, 19 auxiliares e 1 atendente de enfermagem. Como uma das discordâncias, cerca de $80 \%$ do pessoal auxiliar não indicou a necessidade dos itens de higiene serem prescritos para serem efetuados, enquanto $67 \%$ dos enfermeiros indicaram tal necessidade. Como uma das concordâncias, $67 \%$ dos enfermeiros e $85 \%$ do pessoal auxiliar não indicaram a necessidade de prescrever orotraqueal para que seja efetuada. Mudanças no conteúdo da prescrição de enfermagem são sugeridas.
\end{abstract}

ABSTRACT - Upon analysing the practice of what professionals have been doing, we have come to inquire ourselves if the nursing prescription has really been coordinating the nursing practices by attending personel. With the principal objective of knowing and confronting the opinion of the nurses and attending personal concerning the contents of the nursing prescription, we've had 6 nurses, 19 attendants and 1 attending person answer a questionary. As a disagreement, about $80 \%$ of the attending personal didn't mention the necessity of prescribing the hygiene items to be practiced, while $67 \%$ of the nurses indicated such a necessity. As one of the agreements, $67 \%$ of the nurses and $85 \%$ of the attending persórial did not mention the necessity to prescribe grotraqueal aspiration to be practiced. Changes in the contenst on nursing prescription are suggested.

\section{INTRODUÇÃO}

Atualmente, busca-se na enfermagem a melhoria da qualidade de assistência ao paciente, havendo ênfase especial na questão do plane jamento da assistência, com a tentativa de evitar ao máximo as ações de enfermagem rotinizadas e empíricas.

Comenta FRIEDLANDER ${ }^{4}$ que "a assistência tradicional da enfermagem, baseada nas prescrições médicas e na rotina hospitalar, não está satisfazendo aos profissionais, pois, dentro do atual contexto, o paciente começa a ser descaracterizado como ser individual (. . .)".

Ainda a essa respeito SANTOS, cols. ${ }^{7}$ comentam que "a enfermagem sempre esteve preocupada em desenvolver uma metodologia de assistênçia que objetivasse aprimorar a qualidade do cuidado prestado ao paciente. Esta metodologia tem recebido diferentes denominaçōes, como: plano de cuidado, processo de enfermagem, sistemática da assistência ou metodologia de assistência".

Assim, o plane jamento da assistência de enfermagem diz respeito à utilização do método cientifíco na assistência, através de um processo composto por etapas ordenadas, as quais "variam en número e denominação, de acordo com o autor" (CHACCHUR').

Apesar de tais variações e, ainda do referencial teórico adotado pelos diversos autores, a prescrição de enfermagem constitui-se numa das fases do processo de enfermagem.

Para HORTA ${ }^{2}$, a prescrição de enfermagem "é o roteiro diário (ou aprazado) que coordena a ação da equipe de enfermagem nos cuidados adequados ao atendimento das necessidades básicas e específicas do ser humano".

Comenta PAIM" , que "a prescrição de enfermagem significa, portanto, medidas de solução para os problemas de um indivíduo, indi-

\footnotetext{
* Prêmio Noraci Pedrosa Moreira - 20 lugar - 43 Congresso Brasileiro de Enfermagem - Curitiba-PR - 1991

** Enfermeiro do Centro de Terapia Intensiva da Unidade de Emergencia do HCFMRJ-USP.

*** Enfermeiro, mestrando em Enfermagem Fundamental da Escola de Enfermagem de Ribeiräo Preto-USP, Bolsista do CNPa.
} 
cadas e registradas previamente pelo enfermeiro com a finalidade última de atender as necessidades humanas desse mesmo indivíduo sob sua responsabilidade profissional.

Enfocando as unidades de tratamento intensivo acreditamos que a utilização da prescrição de enfermagem possa facilitar o plane jamento dos cuidados, priorizando-os e individualizando-os promovendo a melhoria da qualidade de assistência de enfermagem prestada aos pacientes críticos.

Referem SILVA, FANTINATO ${ }^{8}$ que o enfermeiro além de administrador da unidade deve utilizar seus conhecimentos técnico-científicos, proporcionando aos pacientes cuidados individualizados, (. . .) facilitando um atendimento adequado tanto quantitativa quanto qualitativamente, determinando prioridade, identificando necessidades terapêuticas e valores pessoais do paciente, selecionando recursos indicados para a realização de cuidados".

Nossa experiência profissional em um Centro de Terapia Intensiva que utiliza a prescrição de enfermagem como atividade diária do enfermeiro, vem representando uma fonte de inquietação, que nos remete a uma análise crítica e reflexiva acerca da elaboração da prescrição de enfermagem no referido setor.

A prescrição de enfermagem neste setor $\varepsilon$ utilizada desde o início de suas atividades há cerca de três anos. Porém, não existe definido um referencial teórico que a fundamente.

Ainda, $e ́$ frequente a ocorrência de situações como os elementos da equipe de enfermagem prestarem cuidados ao paciente sem uma leitura prévia da prescrição de enfermagem, ou deixar de cumprí-la em sua totalidade.

Dessa forma, começamos a nos indagar se a prescrição de enfermagem conforme é elaborada pelo denfermeiro vem realmente coordenando os cuidados de enfermagem a serem prestados pelo pessoal auxiliar.

Isso nos leva à hipótese de que o conteúdo da prescrição de enfermagem possa estar atuando como fator desfavorável à sua utilização como instrumento coordenador da assistência de enfermagem

Em vista disso, preocupados em evitar que a prescrição de enfermagem, nesse setor, acabe por sí mesma rotinizada e descaracterizada, nos propomos a realizar este estudo, cujos objetivos são:

1 - Conhecer e confrontar as opiniões dos enfermeiros e do pessoal auxiliar quanto o que representa a prescrição de enfermagem no referido setor;

2 - Conhecer e confrontar as opiniões dos enfermeiros e do pessoal auxiliar com relação à elaboração da prescrição de enfermagem, no que se refere fundamentalmente ao que é prescrito (conteúdo);

3 - Analisar se a prescrição de enfermagem está realmente coordenando as ações de enfermagem;

4 - Sugerir as transformações que forem necessárias com relação ao conteúdo da prescrição de enfermagem.

\section{METODOLOGIA}

O trabalho foi realizado no Centro de Terapia Intensiva da Unidade de Emergência de um hospital-escola de Ribeirão Preto.

O setor é composto por sete leitos, sendo cinco para pacientes adultos e dois para pacientes pediátricos.

Os cuidados de enfermagem são prestados por 8 enfermeiros, 24 auxiliares e 1 atendente de enfermagem.

A amostra em estudo constituiu-se de 6 enfermeiros, 19 auxiliares e 1 atendente de enfermagem, considerando que no período da coleta de dados 1 enfermeira e 2 auxiliares de enfermagem encontravam-se em licença gestante, 3 auxiliares de enfermagem estavam em férias e 1 enfermeira encontrava-se em período de treinamento para admissão.

Considerando que apenas um elemento da amostra é representante da categoria atendente' de enfermagem, as suas respostas serão analisadas juntamente com as respostas dos auxiliares de enfermagem.

A coleta de dados foi feita pelos próprios autores, nos três turnos de trabalho, no período de 27 a 29 de maio de 1991 .

Optamos pela utilização de um questionário (Anexo 1) composto pela identificação da categoria profissional, duas questões e a solicitação de sugestões.

A primeira constituiu-se numa questão aberta a respeito do significado da prescrição de enfermagem no referido setor.

A segunda foi respondida com base nos itens de uma prescrição de enfermagem, ou seja, analisando cada item da prescrição, o elemento da equipe de enfermagem respondeu se o mesmo tem que ser ou não prescrito para que se ja efetuado, justificando a sua resposta.

Antes de iniciar a coleta de dados, o referido instrumento foi colocado em teste (teste-piloto) em outro setor do mesmo hospital que também utiliza a prescrição de enfermagem.

Foi utilizada a prescrição diária de enfermagem de um paciente * que estava recebendo os cuidados da equipe de enfermagem do CTI no período da coleta de dados. Dessa forma, os elementos da equipe de enfermagem puderam responder as questões, baseados na sua realida-

\footnotetext{
* Paciente M.G.S., masculino, 66 a nos, com diagnóstico médico de hipertensảo arterial severa, hemorra gia intra-ventricular, sen-
} do submetido à cirurgia - colocação de derivação ventricular externa (D.V.E.). 
de imediata.

A escolha desse paciente (realizada no primeiro dia da coleta de dados) baseou-se no número de itens da sua prescrição de enfermagem. Assim, foi escolhido o paciente cuja prescrição apresentava o maior número de itens. Tal escolha se deu levando-se em conta que, um dos nossos objetivos, é a análise da opinião dos elementos da equipe de enfermagem quanto ao conteúdo da prescrição, o que justamente se revela através dos itens prescritos.

A cada dia da coleta de dados, foi transcrita a prescrição de enfermagem numa folha à parte, a qual foi entregue aos elementos da equipe de enfermagem para que os mesmos pudessem responder as questões do questionário.

Torna-se importante esclarecer que a prescrição de enfermagem é refeita a cada 24 horas. Assim, com relação à prescrição do mesmo paciente, utilizada em nosso estudo, apreceram três itens novos no decorrer da coleta de dados; ou seja, itens que não constavam na prescrição no primeiro dia da coleta de dados.

Porém, optamos por exclú́-los, visto que tais itens não seriam respondidos por todos os elementos que compõem a amostra em estudo.

Posteriormente, na fase de análise dos dados, foram interpretados os itens que se repeti- ram nas sucessivas prescriçōes e, portanto, serviram de subsídios para as respostas de todos os elementos que compõem a amostra em estudo.

Optamos pela exclusão desses itens, ao invés de retorná-los àqueles que não os haviam respondido, considerando aspectos como: impossibilitaria a obtenção de respostas baseadas na realidade imediata do paciente e o mesmo poderia evoluir para o óbito.

Torna-se importante esclarecer que os resultados obtidos com esse estudo, servirão de subsídios para uma análise crítica da elaboração (conteúdo) apenas da prescrição de enfermagem do paciente adulto desse setor.

\section{RESULTADOS E DISCUSSÕES}

A partir dos dados obtidos através das respostas do pessoal auxiliar de enfermagem e dos enfermeiros do CTI, apresentaremos a análise dos resultados.

Podemos observar através da Tabela 1, alguns dados que julgamos importantes para discussão. Conforme indicado, $8(33,3 \%)$ das respostas indicam que a prescrição de enfermagem no setor representa a especificação das rotinas referentes ao cuidado do paciente.

Tabela 1 - Distribuição numérica e percentual das respostas do pessoal auxiliar quanto o que representa a prescrição de enfermagem no CTI de um hospital-escola de Ribeirão Preto.

\begin{tabular}{l|c}
\hline Resposta & Número (\%) \\
\hline - Rotina & $08(33,3)$ \\
- Lembrete & $05(20,8)$ \\
- Necessária & $02(8,4)$ \\
- Auxf́lio para a execução segura das tarefas & $02(8,4)$ \\
- Não representa nada & $02(8,4)$ \\
- Não respondeu & $02(8,4)$ \\
- Tarefa obrigatória & $01(4,1)$ \\
- Cuidados que mudam de paciente para paciente & $01(4,1)$ \\
- Perda de tempo & $01(4,1)$ \\
\hline Total & $24(100,0)$ \\
\hline
\end{tabular}

Percebemos porém, ao analisarmos as respostas obtidas, diferenças na forma como o pessoal auxiliar aborda a prescrição de enfermagem como rotina. Assim, dentre as 08 respostas, uma indica que a prescrição de enfermagem é importante, apesar de rotineira, visto que tais rotinas incluem cuidados referentes às necessidades básicas do paciente.

Duas respostas do pessoal auxiliar mostram que a maior parte dos itens prescritos representa uma rotina já estabelecida no setor, porém com exceções, como o caso das indicações de curativos e mudanças de decúbito.

As demais respostas referentes à rotina indicam que a prescrição de enfermagem representa apenas rotina.

Dentre as 5 respostas $(20,8 \%)$ que demons- tram que a prescrição de enfermagem representa um lembrete quanto aos cuidados gerais do paciente, percebemos também diferenças na forma pela qual foram abordadas pelo pessoal auxiliar. Assim, pela análise das respostas obtidas, podemos inferir que 3 respostas indicam o lembrete como sendo importante, visto que, os auxiliares de enfermagem tecem comentários como: "a prescrição é bem completa", "a prescrição está correta", ela permite "não esquecer cuidados que possam passar desapercibidos".

Porém, 2 respostas mostram comentários como: "há quem faz sem precisar de prescrição" e ainda, a prescrição de enfermagem é "apenas lembrete".

Conforme demonstrado na mesma tabela, 1 resposta $(4,1 \%)$ indica que a prescrição de en 
fermagem representa as tarefas obrigatórias a serem executadas.

Quanto a essa colocação nós indagamos se são consideradas importantes tais tarefas ou se apenas são executadas por representarem obrigaçōes do setor.

Apesar do percentual pouco significativo, julgamos relevante a discussão das respostas não representa nada e perda de tempo, indicadas pelo pessoal auxiliar. Tais respostas foram relacionadas às rotinas. Uma delas indica que a prescrição de enfermagem é "perda de tempo", por representar rotinas que todo auxiliar de enfermagem já conhece e sabe fazê-las.

Uma outra resposta demonstra que a prescrição de enfermagem "não representa nada", apenas rotinas que devem ser feitas "automaticamente".

COGO, cols. ${ }^{2}$ realizaram um estudo sobre a percepção do pessoal auxiliar quanto à prescrição de enfermagem, em unidade de internação médico-cirúrgicas de um hospital de ensino do Rio Grande do Sul.
Foram obtidas respostas desfavoráveis à utilização da prescrição de enfermagem. Entre elas a justificativa de que os cuidados de enfermagem são automáticos, o fato de serem prescritas rotinas e, ainda o pessoal auxiliar acredita na sua capacidade de avaliar o paciente na prestação dos cuidados.

Muito nos preocupa esse "fazer automático". considerando o paciente crítico do CTI, não julgamos que somente as rotinas possam assistí-los adequadamente, visto que, os cuidados de enfermagem de rotina são entendidos como "cuidados de enfermagem usuais que não exigem especificidade de conhecimento e/ou de tratamento, e que são apresentados a qualquer assistido (geralmente hospitalizado), em horários regulares" (SIMŌES ${ }^{9}$ ).

Como demonstra a Tabela 2, 4 respostas $(57,14 \%)$ dos enfermeiros mostram a prescrição de enfermagem como a determinaçāo dos cuidados necessários ao paciente. Dentre essas respostas uma indica que tais cuidados são determinados de acordo com as necessidades do paciente.

Tabela 2 - Distribuição numérica e percentual das respostas dos enfermeiros quanto o que representa a prescrição de enfermagem no CTI de um hospital-escola de Ribeirão Preto.

\begin{tabular}{c|c}
\hline Resposta & Número (\%) \\
\hline - Determinação dos cuidados necessários ao paciente & $04(57,14)$ \\
- Garantia da execução dos cuidados de enfermagem & $02 \quad(28,57)$ \\
- Alerta para detalhes importantes que possam passar \\
desapercebidos & $01(14,29)$ \\
\hline Total & $07(100,00)$ \\
\hline
\end{tabular}

Compreendemos que este seja um ponto que não condiz com a realidade prática, visto que, como já comentado, no referido setor, não existe definido um referencial teórico que fundamente a prescrição de enfermagem.

Foram obtidas duas respostas $(28,57 \%)$ que indicam que a prescrição de enfermagem representa a garantia da execuçāo dos cuidados de enfermagem. Entendemos que apenas o ato de descrever as ações de enfermagem a serem realizadas, não garante que as mesmas se jam efetuadas, visto que a realização dessas ações depende, dentre outros aspectos, da importância atribuiŕda à prescrição pela equipe de enfermagem e da supervisão pelo enfermeiro.

Ainda, pela análise das respostas dessa tabela, foi obtido que a prescrição de enfermagem representa um alerta para detalhes importantes que possam passar desapercebidos (14,29\%). Apesar desses detalhes terem sido considerados importantes, indagamos se as ações de enfermagem devam ser entendidas como simples detalhes.

ao compararmos as respostas dos enfermeiros e do pessoal auxiliar percebemos que, enquanto 8 respostas $(33,3 \%)$ do pessoal auxiliar indicam a prescrição de enfermagem como rotina, tal resposta não foi mencionada pelos enfermeiros. A maioria das respostas dos enfermeiros $(57,14 \%)$ apontam a prescrição de enfermagem como a determinaçāo dos cuidados necessários ao paciente.

$\mathrm{Na}$ verdade, a prescrição de enfermagem representa, dentre outros aspectos, a determinação das ações a serem efetuadas ao se assistir o paciente. Porém, inferimos que é frequente a prescrição de rotinas pelo enfermeiro, visto que 33,3\% das respostas do pessoal auxiliar assim indicam.

Essa situação pode propiciar a descaracterização da prescrição de enfermagem, o que parece não ter sido percebido pelo enfermeiro, mediante suas respostas.

Em relação à Tabela 3 que mostra a opinião do pessoal auxiliar quanto à necessidade dos itens da prescrição de enfermagem serem prescritos para que sejam efetuados, consideramos alguns aspectos relevantes.

Quanto aos itens referentes às higienes oral, ocular, glande e aplicação de vaselina nos lábios, obtivemos respectivamente $80 \%, 75 \%$, $75 \%$ e $65 \%$ de respostas negativas, ou seja, a 
prescrição de enfermagem não está orientando a execução de tais cuidados.

Percebemos ainda que, na opinião do pessoal auxiliar, os itens observaçāo/avaliaçāo neurológica e aspirar cânula orotraqueal e V.A.S. também não precisam estar prescritos para que os mesmos sejam efetuados, o que é demonstrado pelo significativo percentual en- contrado: $80 \%$ e $85 \%$ respectivamente de respostas negativas.

Quanto aos itens manter roda d'água em regiāo sacral e observar volume e características coletadas na D.V.E., obtivemos $55 \%$ de respostas afirmativas em ambos os itens; o que indica a prescrição de enfermagem está orientando a execução de tais ações.

Tabela 3 - Distribuição numérica e percentual da opinião do pessoal auxiliar do CTI de um hospital escola de Ribeirão Preto, quanto à necessidade de os itens da precrição de enfermagem serem prescritos para que sejam efetuados.

\begin{tabular}{l|rr}
\hline \multicolumn{1}{c|}{ Item } & \multicolumn{2}{c}{ Opinião } \\
\cline { 2 - 3 } & \multicolumn{1}{c}{ Sim (\%) } & Não (\%) \\
\hline - Fazer higiene oral com água mais CepacoI & $04(20)$ & $16(80)$ \\
- Fazer higiene ocular com água boricada 2\% & $05(25)$ & $15(75)$ \\
- Fazer higiene da glande com PVPI Tópico & $05(250$ & $15(75)$ \\
- Aplicar vaselina líquida nos lábios & $07(35)$ & $13(65)$ \\
- Observação/Avaliação neurológica & $04(20)$ & $16(80)$ \\
- Aspirar cânula orotraqueal e V.A.S. & $03(15)$ & $17(85)$ \\
- Manter roda d'água em região sacral & $11(55)$ & $09(45)$ \\
- Observar volume e características coletados & & \\
$\quad$ na D.V.E. & $11(55)$ & $09(45)$ \\
\hline$\quad$ Total & $50(31,25)$ & $110(68,75)$ \\
\hline
\end{tabular}

Através da Tabela 4, analisamos também as respostas dos enfermeiros quanto à necessidade dos itens da prescrição de enfermagem serem prescritos para que sejam efetuados.

Assim, podemos observar que, com relação aos itens referentes às higienes oral, ocular e da glande, obtivemos $67 \%$ de respostas afirmativas para cada um dos itens. Esses resultados se contrapōem aos resultados obtidos com o pessoal auxiliar.
Quanto ao item observaçāo/avaliaçāo neurologica a mesma tabela indica que as opiniōes se dividiram, ou seja obtivemos $50 \%$ de respostas afirmativas e $50 \%$ de respostas negativas. Este resultado nos possibilita inferir que a prescrição desse item se rotinizou a tal ponto que mesmo aqueles que não julgam necessária a sua prescrição, no dia-a-dia, continuam incluindo tais itens em sua prescrição.

Tabela 4 - Distribuição Númerica e Percentual da Opinião dos Enfermeiros do CTI de um Hospital Escola de Ribeirão Preto Quanto à Necessidade de os Itens da Prescrição de Enfermagem serem Prescritos para que sejam efetuados.

\begin{tabular}{l|rr}
\hline \multicolumn{1}{c|}{ Item } & \multicolumn{2}{c}{ Opiniāo } \\
\cline { 2 - 3 } & Sim (\%) & Não (\%) \\
\hline - Fazer higiene oral com água mais Cepacol & $04(67)$ & $02(33)$ \\
- Fazer higiene ocular com água boricada 2\% & $04(67)$ & $02(33)$ \\
- Fazer higiene da glande com PVPI Tópico & $04(67)$ & $02(33)$ \\
- Aplicar vaselina líquida nos lábios & $05(83)$ & $01(17)$ \\
- Observação/Avaliação neurológica & $03(50)$ & $03(50)$ \\
- Aspirar cânula orotraqueal e V.A.S. & $02(33)$ & $04(67)$ \\
- Manter roda d'água em região sacral & $05(83)$ & $01(17)$ \\
- Observar volume e características coletados & & \\
$\quad$ na D.V.E. & $05(83)$ & $01(17)$ \\
\hline$\quad$ Total & $32(67)$ & $16(33)$ \\
\hline
\end{tabular}

Com relação ao item aspirar a cânula orotraqueal e V.A.S., $67 \%$ dos enfermeiros responderam negativamente quanto à necessidade do mesmo ser prescrito para que seja efetuado, o que se constitui num ponto de concordância com as respostas do pessoal auxiliar.

Ainda, quanto aos itens aplicar vaselina líquida nos lábios, manter roda d'água em regiāo sacral e observar volume e características coletadas na D.V.E., obtivemos 
83\% de respostas afirmativas para cada um dos itens.

Ao compararmos tais resultados com as respostas do pessoal auxiliar, percebemos que o item aplicar vaselina líquida nos lábios, constitui-se um ponto de discordância. Por sua vez, os itens observar volume e características coletados na D.V.E. e manter roda d'água em regiâo sacral, indicam pontos de concordância com o pessoal auxiliar, apesar do maior percentual das respostas dos enfermeiros.

A Tabela 5 mostra as justificativas do pessoal auxiliar quanto à necessidade dos itens da prescrição de enfermagem serem prescritos para que se jam efetuados.

Nos itens da prescrição, exceto higiene ocular e aspirar a cânula orotraqueal, obtivemos como justificativa predominante para a resposta afirmativa o lembrete, o que pode ser visualizado pelo percentual indicado na mesma tabela.

No nosso entender, lembrar a alguém o que deve ser feito, pode talvez, representar que estamos apenas evitando que o outro esqueça o que já sabe. Porém, nos indagamos, se o que o pessoal auxiliar já sabe refere-se às rotinas do setor ou aos cuidados que individualizam o paciente.

Quanto a justificativa pode variar de paciente para paciente, notamos que a mesma foi inil licada em todos os itens, exceto no item fazer higiene da glande. Porém, nota-se percentual significativo apenas nos itens higiene oral, higiene ocular, manter roda d'água e observar caracterfsticas da D.V.E.

Analisando as justificativas das respostas negativas, podemos observar que as predominantes foram rotina e sabemos o que deve ser feito.

Quanto à justificativa rotina a mesma foi mais citada em todos os itens da prescrição, exceto nos itens aplicar vaselina nos lábios e aspirar a cannula orotraqueal e V.A.S., nos quais a justificativa mais citada foi sabemos $o$ que ser feito, o que é verificado pelo percentual indicado na mesma tabela.

Observando o percentual obtido quanto à justificativa rotina, e comentamos que, provavelmente, para o pessoal auxiliar não é a prescrição de enfermagem que coordena a execução das ações de enfermagem, mas, talvez a própria rotina de trabalho estabelecida no setor.
Porém, parece-nos que, algumas vezes :o pessoal auxiliar não consegue visualizar dentre as chamadas rotinas, o que na verdade não deveria ser assim considerado. Isso pode ser confirmado ao observamos o percentual obtido com relação à rotina no item manter a roda d'água na regiāo sacral (45\% do pessoa auxiliar acredita que tal item não precisa ser prescrito para que seja ef etuado e a maioria (25\%) aponta como justificativa a rotina), já que esse item, na nossa opinião não deveria ser entendido como rotina, visto que depende, primordialmente, da avaliação de cada paciente.

Podemos observar que a justificativa sabemos o que deve ser feito, representada por $\mathbf{4 4 , 4 \%}$ das justificativas das respostas negativas, a aproxima do maior percentual encontrado (rotina-46,2\%).

Acreditamos que, dentre o pessoal auxiliar, existem elementos que sabem avaliar o paciente, indicando alguns cuidados que o mesmo necessita. Porem, quando tais pessoas afirmam que alguns itens como, aplicar vaselina líquida nos lábios e manter roda d'água em regiāo sacral não precisam ser prescritos para serem efetuados; parece-nos que as mesmas não conseguem ter a visão da continuidade da assistência, ou seja, como garantir uma assistência adequada durante as $24 \mathrm{~h}$, considerando que não é uma única pessoa que cuida do paciente ?

Quanto aos itens aspirar a cânula orotraqueal e VAS e fazer observaçāo/avaliaçāo neurológica, observamos um percentual significativo quanto à justificativa sabemos o que deve ser feito, o que e representado por $75 \%$ e $35 \%$ respectivamente.

Na nossa opinião, tais itens são relevantes e mesmo vitais para o paciente $e$, numa situação ideal o pessoal auxiliar deve fazê-los, por compreender as complicações advindas da não execução dos mesmos.

Infelizmente não podemos, na prática, fazer essa afirmação com total segurança, visto que, trabalhamos com pessoas distintas quanto valores pessoais, conhecimentos e senso de. responsabilidade. Porém, acreditamos que não é a prescrição de enfermagem que garante a execução desses cuidados, como já mencionado e, sim um processo de educação continuada e supervisão exercidos pelo enfermeiro. 
Tabela 5 - Distribuição numérica e percentual das justificativas do pessoal auxiliar do CTI de um hospital escola de Ribeirão Preto quanto à necessidade de os itens da prescrição de enfermagem serem prescritos para que sejam efetuados

\begin{tabular}{|c|c|c|c|c|c|c|c|c|c|c|c|c|c|}
\hline \multirow{2}{*}{$\begin{array}{ll}\text { Item } & \text { Justificativa } \\
\end{array}$} & \multicolumn{6}{|c|}{$\mathrm{S}$ i m } & \multicolumn{7}{|c|}{ Não } \\
\hline & $\mathrm{A}(\%)$ & $\mathrm{B}(\%)$ & $\mathrm{C}(\%)$ & $\mathrm{D}(\%)$ & $\mathrm{E}(\%)$ & Total & $\mathrm{F}(\%)$ & $\mathrm{G}(\%)$ & $\mathrm{H}(\%)$ & I( & $\mathrm{J}(\%)$ & $\mathrm{K}(\%)$ & Total \\
\hline $\begin{array}{l}\text { - Fazer higiene oral com água } \\
\text { mais Cepacol }\end{array}$ & $1(5)$ & $2(10)$ & $1(5)$ & & & & $11(55)$ & $4(20)$ & $1(5)$ & & & & \\
\hline $\begin{array}{l}\text { - Fazer higiene ocular com água } \\
\quad \text { boricada } 2 \% \\
\text { - Fazer higiene da glande com }\end{array}$ & & $2(10)$ & $1(5)$ & $1(5)$ & $1(5)$ & & $8(40)$ & $5(25)$ & $2(10)$ & & & & \\
\hline PVPI Гб́pico & $3(15)$ & & & $1(5)$ & $1(5)$ & & $10(50)$ & $4(20)$ & $1(5)$ & & & & \\
\hline $\begin{array}{l}\text { - Aplicar vaselina nos labios } \\
\text { - Observação/Avaliação }\end{array}$ & $4(20)$ & $1(5)$ & & & $2(10)$ & & $4(20)$ & $6(30)$ & 1(5) & $2(10)$ & & & \\
\hline $\begin{array}{l}\text { neurrológica } \\
\text { - Aspirar cânula orotraqueal }\end{array}$ & $2(10)$ & $1(5)$ & & & $1(5)$ & & $8(40)$ & $7(35)$ & & & $1(5)$ & & \\
\hline $\begin{array}{l}\text { e V.A.S. } \\
\text { - Manter roda d'água em região }\end{array}$ & & $1(5)$ & & & $2(10)$ & & $1(5)$ & $15(75)$ & $1(5)$ & & & & \\
\hline $\begin{array}{l}\text { sacral } \\
\text { - Observar volume e caracterís- }\end{array}$ & $6(30)$ & $3(15)$ & & & $2(10)$ & & $5(25)$ & $4(20)$ & & & & & \\
\hline ticas c & $6(30)$ & $5(25)$ & & & & & $5(25)$ & $3(15)$ & & & & $1(5)$ & \\
\hline Total & $22(44)$ & $15(30)$ & $2(4)$ & $2(4)$ & $9(18)$ & 50 & $52(46,2)$ & $8(44,4)$ & $6(5,5)$ & $2(1,8)$ & $1(0,9)$ & $1(0,9)$ & 110 \\
\hline
\end{tabular}

Legendas: SIM

$\mathrm{A}=$ Lembretes

$\mathrm{B}=$ Pode variar de paciente para paciente

$\mathrm{C}=$ Apesar de rotina devo ser prescrito

$\mathrm{D}=$ Em casos de admissão

$E=$ Não justificou
Nāo

$\mathrm{F}=$ Rotina

$\mathrm{G}=$ Sabemos o que deve ser feito

$\mathrm{H}=$ Tarefa obrigatória

$\mathrm{I}=$ Pode variar de paciente para paciente

$\mathrm{J}=$ É prescrito pelo médico

$\mathrm{K}=$ Compete ao médico

A Tabela 6 mostra as justificativas dos enfermeiros quanto à necessidade de os itens da prescrição de enfermagem serem prescritos para que sejam efetuados.

Tabela.6 - Distribuição numérica e percentual das justificativas dos enfermeiros do CTI de um hospital escola de Ribeirão Preto quanto à necessidade de os itens da prescrição de enfermagem serem prescritos para que sejam efetuados.

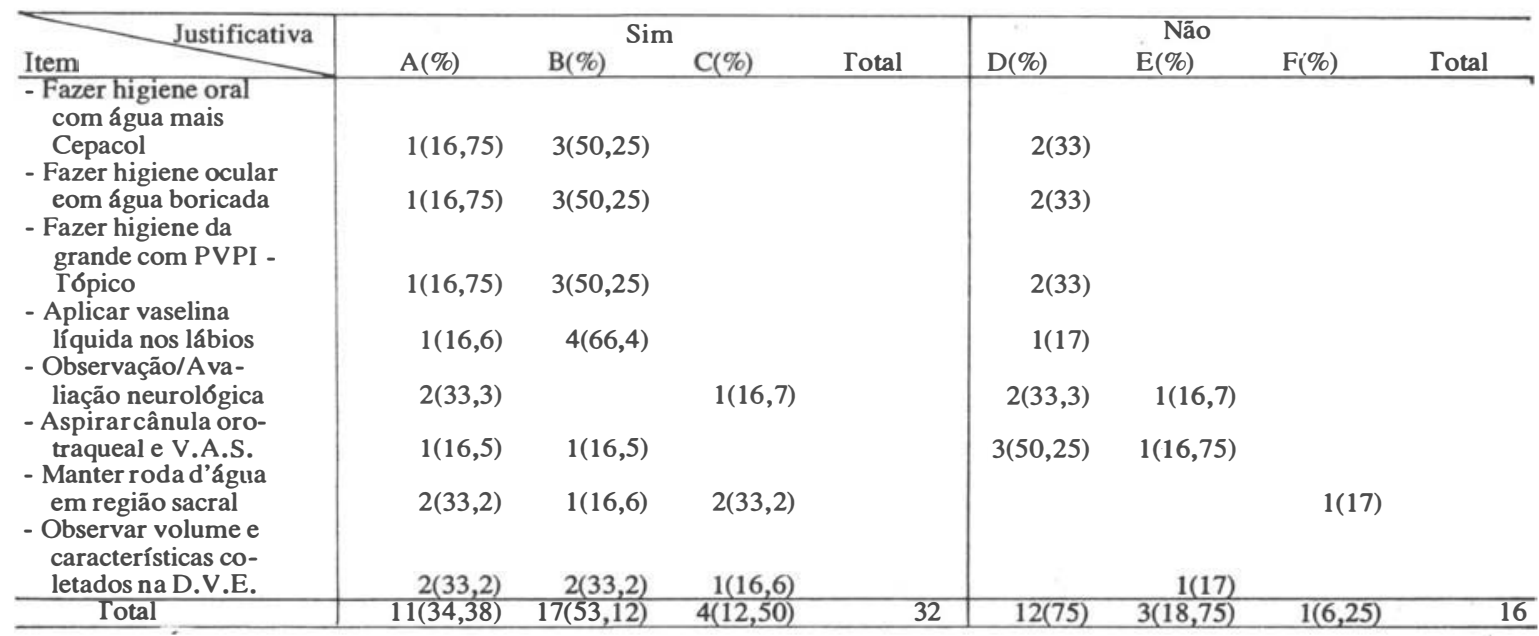

\section{Legenda: SIM}

$A=$ Particulariza o paciente

B = Garantia para ser efetuado

$\mathrm{C}=$ lembrete

\section{NĀO}

$\mathrm{D}=$ Rotina

$E=A$ equipe sabe o que deve ser feito $\mathrm{F}=$ Desde que se introduza uma rotina no procedimento 
Quanto às justificativas das respostas afirmativas, a mais indicada foi garantia para ser efetuado $(53,12 \%)$ das justificativas), a qual aparece em todos os itens, exceto observaçāo/avaliaçāo neurologica. Nos itens referentes à higiene e aplicação de vaselina nos lábios, essa justificativa aparece com um percentual bem significativo: $50,25 \%$ dos enfermeiros justificaram que a prescrição dos itens referentes às higienes oral, ocular e da glande, garante a execução dos mesmos. Quanto ao item aplicar vaselina líquida nos lábios, $66,4 \%$ dos enfermeiros indicam a mesma justificativa.

Ao compararmos os resultados referentes à higiene entre os enfermeiros e o pessoal auxiliar, notamos um ponto de discordância. A maioria do pessoal auxiliar (indicado pelo percentual na Tabela 5) mostra que tais itens são rotinas e, portanto, a sua execução não depende da prescrição de enfermagem. Por sua vez, a maioria dos enfermeiros (o que $\varepsilon$ indicado na Tabela 6) mostra que tais itens precisam ser prescritos, no sentido de garantia de execução.

$\mathrm{Na}$ nossa opinião, a prescrição de enfermagem deve ser utilizada, primordialmente, como forma de individualizar o cuidado do paciente.

Assim, CHACCHUR ${ }^{1}$ citando COLLINGWOOD ${ }^{3}$ comenta: "o plano de cuidados é um instrumento utilizado para auxiliar na individualização do cuidado do paciente, (. . .). Constitui um "roteiro" das ordens, ou prescrições de enfermagem (....)".

A Tabela 6 indica que a justificativa particulariza o paciente foi citada em todos os itens da prescrição, porém com percentual mais significativo nos itens observaçāo/avaliaçāo neurológica (33,3\%), manter roda d'água em regiāo sacral $(33,2 \%)$ e observar volume e características coletados na
D.V.E. $(33,2)$.

Entretanto, ao observarmos o percentual total obtido com a justificativa particulariza o paciente $(34,38 \%)$, notamos que a mesma vem seguida à justificativa garantia para ser efetuado $(53,12 \%)$. Tal situação nos mostra que a prescrição de enfermagem está sendo utilizada, principalmente, com o objetivo de garantir as ações executadas, talvez sendo estas ações que particularizam o paciente ou rotinas.

A justificativa lembrete foi, conforme indicado na tabela, a menos citada (ao contrário do resultados obtidos com o pessoal auxiliar). Mas, teve um percentual significativo $(33,2 \%)$ no item manter roda d'água sacral.

Tal resultado nos incomoda, visto que, acreditamos que tal ítem deve ser prescrito com o intuito de particularizar o paciente.

Quanto às justificativas das respostas negativas dos enfermeiros obtivemos como a mais indicada a rotina $(75 \%)$.

Essa justificativa teve percentual significativo nos itens referentes à higiene (33\% para cada um dos itens - higiene oral, ocular e da glande) e observação neurológica $(33,3 \%)$ e predominantemente no item aspirar a cânula orotraqueal $(50,25 \%)$.

Tornar-se relevante analisarmos que, para o item aspirar cânula orotraqueal a VAS, a justificativa das respostas negativas mais citada pelo pessioal auxiliar foi sabemos o que deve ser feito (75\%); enquanto para os enfermeiros a justificativa a equipe sabe o que deve ser feito obteve somente o percentual $16,75 \%$.

Através do Quadro 1, podemos visualizar algumas sugestōes do pessoal auxiliar e dos enfermeiros do"CTI, quanto à elaboração da prescrição de enfermagem.

Quadro 1 - Sugestões do pessoal auxiliar e enfermeiros do CTI de um hospital-escola de Ribeirão Preto quanto à elaboração da prescrição de enfermagem.

\begin{tabular}{|l|l|}
\hline \multicolumn{1}{|c|}{ Pessoal Auxiliar } & \multicolumn{1}{c|}{ Enfermeiros } \\
\hline - Não prescrever rotinas; & - Não prescrever rotinas; \\
- Avaliar o paciente primeiramente; & - Dar continuidade às prescrições; \\
- Avaliar as condições do plantão ao elaborar a a & - Prescrever itens relacionados ao relaciona- \\
prescrição; & mento do pessoal de enfermagem com o pacien- \\
- Prescrever em uma folha única para enferma- & - Não se restringir apenas em cuidados de roti- \\
gem; & na; \\
- Prescrever somente as novas rotinas. & - Incluir itens como: \\
& $\bullet$ avaliação dos parâmetros vitais antes de ad- \\
& ministrar medicações \\
& $\bullet$ Observação de efeitos colaterais \\
& $\bullet$ Avaliar estado emocional do paciente. \\
\hline
\end{tabular}




\section{CONCLUSÕES}

Com o propósito de retornarmos aos resultados que julgamos mais significativos; elaboramos uma síntese das opiniões dos enfermeiros e do pessoal auxiliar quanto à necessidade da espeficicação de cada um dos itens da pres- crição de enfermagem em estudo, para que os mesmos sejam efetuados, com a respectiva justificativa. Para a elaboração dessa síntese nos baseamos nas respostas com percentuais mais significativos.

A síntese está demonstrada através dos quadros abaixo:

Justificativas do Pessoal Auxiliar

\begin{tabular}{|c|l|l|}
\hline Item & Sim & Não \\
\hline 1 & & Rotina \\
2 & & Rotina \\
3 & & Rotina \\
4 & & Sabemos o que deve ser \\
& & efetuado \\
5 & & Rotina \\
6 & & Sabemos o que deve ser \\
7 & & efetuado \\
8 & Lembrete & \\
\hline
\end{tabular}

Concluímos que para o pessoal auxiliar a prescrição de enfermagem em estudo está apenas coordenando a execução dos itens observar volume e características coletados na DVE (item 7) e manter rota d'água em região sacral (item 8); apesar da justificativa mais mencionada ter sido o lembrete.

Para os enfermeiros, a mesma prescrição está coordenando quase a totalidade dos itens prescritos, exceto no item aspirar a cânula orotraqueal e VAS (item 6) e, no item 7 - observação/avaliação neurológica as opiniōes de dividiram.

Dessa forma, para o pessoal auxiliar a prescrição de enfermagem é entendida, predominantemente, como a determinação de rotinas do setor; enquanto, para os enfermeiros a prescrição de enfermagem está sendo utilizada, principalmente, para garantir a execuçāo dos cuidados de enfermagem.

Ao prescrever rotinas, o enfermeiro acaba por descaracterizar a prescrição de enfermagem, a qual passa a ser representada pela maioria do pessoal auxiliar, unicamente, como a especificação das rotinas necessárias ao paciente.

Comp eendemos que seja fundamental a determinação de critérios para se estabelecer, no setor, quais os cuidados de enfermagem que realmente devem ser entendidos como rotineiros.

Sugerimos que, após a determinação dos cuidados de rotina, os mesmos se jam registrados na folha na qual são feitas as anotações de enfermagem ou então que se crie um impresso tipo "check-list" para tal. E, assim a prescrição de enfermagem deverá incluir apenas os cuidados que individualizam o paciente.
Justificativa dos Enfermeiros

\begin{tabular}{|c|l|l|}
\hline Item & \multicolumn{1}{|c|}{ Sim } & Não \\
\hline 1 & Garantia para ser efetuado & \\
2 & Garantia para ser efetuado & \\
3 & Garantia para ser efetuado & \\
4 & Garantia para ser efetuado & \\
5 & Particulariza o paciente & Rotina \\
6 & & Rotina \\
7 & Particulariza o paciente/Lembrete & \\
8 & Particulariza o paciente e garantia & \\
& para ser efetuado & \\
\hline
\end{tabular}

Desse modo, enfocando a prescrição de enfermagem em estudo acreditamos que os itens aplicar vaselina nos lábios e manter roda d'água em região sacral devem ser prescritos, por individualizar o paciente.

Porem, quanto aos itens de higiene, a decisão de prescrevê-los ou não deve ficar na dependência da reflexão sugerida acercà dos cuidados de rotina.

É importante que o enfermeiro do setor busque conhecimentos relacionados à metodologia de assistência, bem como conhecimentos relacionados às próprias condutas de enfermagem.

Sugerimos que os enfermeiros do setor adotem um referencial que fundamente a prescrição de enfermagem.

É ainda importante que tais conhecimentos sejam adequados à realidade na qual se desenvolve a prática de enfermagem. Assim, no setor em estudo, a observação neurológica e a aspiração da cânula orotraqueal constituem açōes vitais para o cuidado do paciente, não havendo a necessidade de serem prescritas para que sejam efetuadas.

Sugerimos a necessidade de outros estudos para a análise crítica de itens que não foram abordados, pela prescrição de enfermagem em estudo.

Consideramos, ainda, que seja importante que enfermeiros e auxiliares de enfermagem reflitam juntos essa temática. É claro, que o ato de prescrever $\epsilon$ direito legal do enfermeiro, mas, isto não impede que o pessoal auxiliar participe de algumas decisões. Afinal, a participação gera o compromisso. 


\section{REFERÊNCIAS BILIOGRÁFICAS}

1 CHACCUR, M.I.B. Análise da implantação do plane jamento da assistência de enfermagem em unidades de internação de um hospital de ensino، Rev. Bras. Enf., Brasilia, 37(3/4):218-227, jul./dez., 1984.

2 COGO, A.L.P. \& cols.. Prescrição; percepção do pessoal auxiliar de enfermagem de unidade médico-cirúrgicas em um hospital de ensino. Rev. Bras. Enf., Porto Alegre, 7(1):61-77, jan., 1986.

3 COLLINGWOOD, M.P. The nursing care plan as a basis for an information system based upon individualised patient care. Nurs. Times, London, 71(12) suppll. 21-2, mar. 20, 1975. In: CHACCUR, M.I.B. - Análise da implementação do planejamento da assistência de enfermagem em unidades de internação de um hospital de ensino. Rev. Bras. Enf., Brasília, 37(3/4):218-227, jul./dez., 1984.

4 FRIENDLANDER, M.R. O processo de enfermagem ontem, hoje e amanhã. Rev. Bras. Enf. USP, São Paulo, 15(2):129-134. 1981 .
5 HORTA, W.A. Processo de enfermagem. São Paulo: EPU/EDUSP, 1979

6 PAIM, L. Plano assistencial e prescriçōes de enfermagem. Rev. Bras. Enf., Braślia, 29:66-82, 1976.

7 SANTOS, L.C.R. et alii. O ensino da metodologia da assistência de enfermagem: responsabilidade da disciplina de Fundamentos de Enfermagem. Rev. Bras. Enf. USP, São Paulo, 21(1):75-88, 1987.

8 SILVA, A.B.F., FAN TINA TO, J.C. Introdução do Planejamento de Cuidados de Enfermagem no Centro de Terapia Intensiva. .Rev. Gaúcha de Enf., Porto Alegre, 4(2):189-199, jul., 1983.

9 SIMŌES, C. Contribuiçāo ao estudo da terminologia básica de enfermagem no Bravil - Taxionomia e Conceituação. Ribeirão Preto, v. 3, 1980 (Tese - Doutorado). 